\title{
Myotonic Dystrophy Confirmed after Cesarean Section
}

\author{
Seung Hyun Kim, Jeongmin Kim, Taehoon Ha, and Sungwon Na \\ Department of Anesthesiology and Pain Medicine, Anesthesia and Pain Research Institute, Yonsei University College of Medicine, Seoul, Korea
}

Dear Editor:

We report a case of Myotonic dystrophy (MD) confirmed after cesarean section under general anesthesia. MD is a common neuromuscular disease in adults, and is characterized by myotonia, and myopathy of the voluntary and involuntary muscles. Patients with MD are at high risk during general anesthesia.

A 33-year-old pregnant woman with hydramnios was admitted to the delivery room, and emergency cesarean section was performed under general anesthesia using $40 \mathrm{mg}$ of rocuronium. After the surgery, the anesthesiologist confirmed partial neuromuscular blockade by train-of-four electrical stimulation and neostigmine $1 \mathrm{mg}$ was used for reversal of neuromuscular blocking agents. However, as the patient's self-respiration was continuously weak, sugammadex $200 \mathrm{mg}$ was additionally administered and then the patient was extubated. In the postanesthesia care unit, she was alert and cooperated well, but presented with shallow breathing continuously; and arterial blood sampling revealed respiratory acidosis, hypoventilation, and hypercapnia. Due to diminished consciousness, reintubation was performed, and the patient was admitted to the intensive care unit (ICU). On both the second and third day after ICU admission, she met the spontaneous breathing trial (SBT) safety criteria respectively which consists of inspiratory pressure $<-25 \mathrm{cmH}_{2} \mathrm{O}$, tidal volume $>5 \mathrm{~mL} / \mathrm{kg}$, vital capacity $>10 \mathrm{~mL} / \mathrm{kg}$, minute ventilation $<10 \mathrm{~L} / \mathrm{min}$, and rapid shallow breathing index $<100$. Extubations was performed on the second and third day post admission, but each time we had to reintubate the patient because of consistent hypercapnia, shallow breathing, and sequential desaturation within several hours.

For diagnosis, we obtained medical history about the patient's shallow breathing pattern. Her husband reported that she had "a feeling of weakness" and exhibited upper eyelid drooping in the evening during the third trimester of pregnancy. Gene analysis performed on the seventh day after ICU admission revealed expansion of
Received on October 12, 2016

Revised on November 22, 2016

Accepted on November 23, 2016

\section{Sungwon $\mathrm{Na}$}

Department of Anesthesiology and Pain Medicine, Anesthesia and Pain Research Institute, Yonsei University College of Medicine, 50 Yonsei-ro, Seodaemun-gu, Seoul 03722, Korea

Tel: +82-2-2227-4237

Fax: +82-2-312-7185

E-mail: NSWKSJ@yuhs.ac

*No potential conflict of interest relevant to this article was reported. 
cytosine-thymine-guanine (CTG) repeats of the dystrophia myotonica protein kinase gene, which could confirm the diagnosis of MD. After the diagnosis of MD, tracheostomy was performed, and she was transferred to the general ward for respiratory rehabilitation treatment.

Our patient did not have a family history of MD and did not present with definite symptoms of muscle wasting. During the preoperative period, the possibility of MD was overlooked, and surgery was performed under general anesthesia. However, after the repeated weaning failure, we could diagnose MD based on the husband's statement, electromyographic studies, and gene analysis.

The MD gene is located on the long arm of chromosome 19 band 13 q. It codes a protein kinase found in skeletal muscle, where it possibly plays a regulatory role. This gene contains an unstable tri-nucleotide repetition CTG. In normal subjects, the number of repeats ranges from 5 to 34; however, in patients with MD, the CTG pattern is repeated as many as 2,000 times, disrupting the normal function of the protein [1]. Individuals with a repeat size between 35 and 49, designated permutation status, or mutable normal are asymptomatic. A mutation of 50 to approximately $150 \mathrm{CTG}$ repeats is associated with a mild disease phenotype, typically characterized by cataracts and mild myotonia. Age at onset is between 20 and 70 years, and life expectancy is normal or moderately reduced. Longer repeats, in the range of 100 to 1,000 , are seen in individuals with the classic MD phenotype (onset between 12 and 30 years of age) with muscle wasting and weakness, myotonia, cataracts, and cardiac conduction defects [2-4].

Pregnancy is associated with an exacerbation of this myopathy. Major antepartum complications include polyhydramnios, preterm labor, urinary tract infections, and placenta previa. Polyhydramnios of unknown etiology may be the first sign of MD. In our case, genetic analysis revealed 73 CTG repeats, which corresponds to the mild disease phenotype. If MD is diagnosed preoperatively, regional anesthesia may be recommended for cesarean section because patients with MD are particularly sensi- tive to anesthetics, and intra- and postoperative complications are relatively frequent. However, when general anesthesia is required, monitoring of neuromuscular function appears to be safe and is important in the management of these patients.

In case of a failed SBT, it is important to take a careful history of patient symptoms, and appropriate examination should follow. In patients with neuromuscular disease, trial extubation can be performed when standard readiness criteria are met. In patients in whom extubation is unsuccessful, timely progression to tracheostomy placement can be considered.

ORCID

Jeongmin Kim http://orcid.org/0000-0002-0468-8012

Sungwon $\mathrm{Na} \quad$ http://orcid.org/0000-0002-1170-8042

\section{References}

1. Khan ZA, Khan SA. Myotonic dystrophy and pregnancy. J Pak Med Assoc 2009; 59: 717-9.

2. Harley HG, Rundle SA, MacMillan JC, Myring J, Brook JD, Crow S, et al. Size of the unstable CTG repeat sequence in relation to phenotype and parental transmission in myotonic dystrophy. Am J Hum Genet 1993; 52: 1164-74.

3. Dalton JC, Ranum LP, Day JW. GeneReview(R) [Internet]. Seattle (WA): University of Washington; 2006. Chapter 454, Myotonic Dystrophy Type 2; [cited 2006 Sep 21]; [about 26 screens]. Available from: https://www.ncbi.nlm.nih.gov/books/ NBK1466/.

4. Arsenault ME, Prévost C, Lescault A, Laberge C, Puymirat J, Mathieu J. Clinical characteristics of myotonic dystrophy type 1 patients with small CTG expansions. Neurology 2006; 66: 1248-50. 